\title{
Low Power and Bluetooth-Based Wireless Sensor Network for Environmental Sensing Using Smartphones
}

\author{
Siamak Aram, Amedeo Troiano, Francesco Rugiano, and Eros Pasero \\ Department of Electronics and Telecommunications, Politecnico di Torino, Torino, Italy \\ \{siamak.aram, amedeo.troiano, francesco.rugiano, \\ eros.pasero\} @polito.it
}

\begin{abstract}
Current research and improvements in the field of wireless sensor networks are focused on decreasing the power consumption and miniaturization, improved smartness and better wearability of the sensor, and especially with their capability for environmental sensing. Today, the survival of these kinds of networks is a critical issue especially in order to keep environmental information updated. This paper presents, an improvement of the environmental sensing acquisition system shown in [1], by applying more sensors to gather data. It was found a novel method of reading sensor data using smartphones and also the structure of sensors themselves helps to decrease the power consumption of the network.
\end{abstract}

Keywords: Wireless Sensor Networks, Bluetooth based sensors, Environment Sensing, Smartphones, Low Power consumption.

\section{Introduction}

In 1980s, the first real mobile phone was neither light nor small, and the problem of power consumption, which still remains today, created difficulty for working in Wireless Sensor Networks (WSN). The technological evolution from the first generation of mobile phones has shown swift growth in different aspects. This development started from the very low-end mobile phone category [2] up to smartphones today. Multitasking operating systems, running myriad of applications and having several features (such as Bluetooth) [3] are the attributes that make these types of phones important in daily life and consequently make them one of the mostused electronic devices. Now, they do more than just connecting people to each other, and have various applications, such as health and medical [4], military, environmental, home and office [5], and commercial. The potential for computing, communicating, analyzing, and monitoring by these new generations of mobile phones allows an increase in quality of life by gathering and scrutinizing environmental information. Traditional environmental monitoring systems have been changed by the new features that WSNs offer.

In some applications, wireless sensor networks use sensors placed in pre-planned locations or equipped on carriers or vehicles, with fixed routing strategies; on the other hand, currently, the capabilities of mobile devices, especially smartphones, as 
data collectors make it possible to distribute sensors, and helps to share information with communities, that is crucial for making decisions for different communities such as governments, groups of people, and researchers [6].

In [1], a smartphone used applied to get environmental information from small spaces, using a Bluetooth transceiver. As shown in Fig. 1A, the approach described in [1] was to acquire temperature and humidity values (considering low cost and low power components) with a Bluetooth communication system for the transmission of the acquired data to an android-based smartphone. The system was tested in a climate chamber that changes the environmental conditions. This scenario makes it easier to get environmental information while moving; moreover, it makes control and warning possible in different fields, such as medical, social services, and agriculture. Bluetooth is one of the most important features in all smartphones that enables both sensor and phone to communicate and transfer data at short distances using less power than Wi-Fi [7].

A)

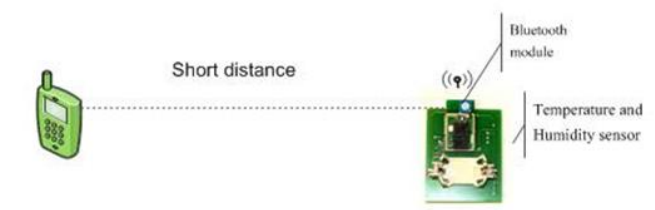

Smart Phone

Bluetooth-based sensor

B)

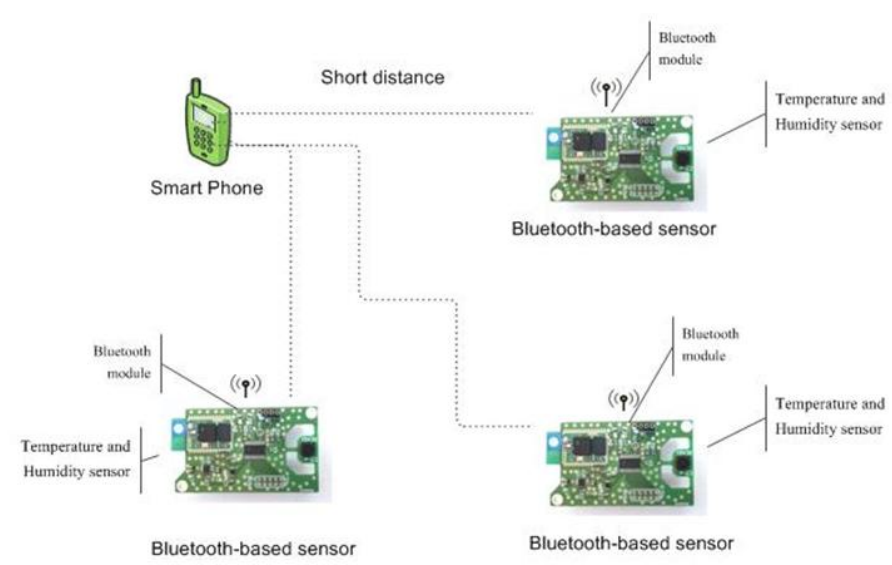

Fig. 1. Acquisition of the environmental data using a smartphone. A) Approach described in [1], direct connection between the smartphone and a sensor. B) Approach of this work, connection between the smartphone and several sensors.

As shown in Fig. 1B, a new scenario was introduced by applying several sensors with different structure. In fact, increasing the number of distributed sensors maximizes the lifetime of the network, since more failures can be tolerated; this tackles the power consumption challenge. Another advantage of applying more 
sensors at the same time is an increased reliability of the network. The smartphone application manages the sampling frequency and the method of connection between sensors using an independent communication with each sensor, without the need for multi-hop routing [8] to gather environmental information. The data is gathered and analyzed directly by the smartphone application.

In this paper, we focus on two points. Firstly, a new device for the acquisition of environmental parameters with consideration of low power consumption was developed. Secondly, the android application presented in [1] was improved with the possibility of acquiring data from a number of sensors at the same time.

\section{Methods}

\subsection{Bluetooth-Based, Low Power Temperature and Humidity Acquisition System}

A Bluetooth based temperature and humidity acquisition system was presented in [1]. That device is battery powered and comprises a temperature and humidity sensor, and a microcontroller that transmits these climatic parameters to a receiver using a Bluetooth module. However, the presented device consumes too much since low power techniques were not adopted during the design; consequently, the battery lifetime is limited. In order to reduce power consumption, a novel Bluetooth based temperature and humidity acquisition system was designed. A low power temperature and humidity sensor (SHT21 from Sensirion, temperature range from -40 to $+125^{\circ} \mathrm{C}$ and accuracy of $0.3^{\circ} \mathrm{C}$, humidity range from 0 to $100 \%$ and accuracy of $2 \%$ ) was used to sense environmental parameters. The sensor communicates the environmental parameters to a microcontroller (MSP430 from Texas Instrument) using the $\mathrm{I}^{2} \mathrm{C}$ protocol. This microcontroller was selected because of its low power consumption and its integrated peripherals. In fact it consumes on average $24 \mu \mathrm{W}$ when transmitting data each minute, while the one used in the previous device consumes about $3 \mathrm{~mW}$. In order to avoid power consumption, the microcontroller switches on the temperature and humidity sensor only during the acquisition of the environmental parameters, then it switches it off. Moreover, the microcontroller puts itself in a low power mode between two consecutive measurements in order to save power. The time distance between two consecutive temperature and humidity measures can be set directly by the user; since the power consumption is proportional to the measuring frequency, modifying this parameter the user can select the best tradeoff between high sampling rate and long battery life. Bluetooth was chosen for its relatively low power consumption (compared with other high data rate wireless communication systems, such as Wi-Fi [7]), simplicity, wide use in the world, the capability to work in lack of particular conditions (e.g. the absence of the Wi-Fi connection), and mainly because it is embedded in nearly all smartphones. The Bluetooth module F2M03GLA from Free2Move was chosen for the designed device. Again, the main criterion used in this choice was power consumption; in fact it consumes about $26 \mathrm{~mW}$ when it is waiting for connection and $90 \mathrm{~mW}$ during the transmission. The Bluetooth module receives data from the UART interface of the microcontroller, and forwards it to a receiver 
using the SPP (Serial Port Profile) service. The microcontroller switches the Bluetooth module on only during the transmission of the environmental parameters, and then turns it off. In order to reduce the power consumption at minimum, switching off the Bluetooth module was preferred to putting it in sleep mode. The device is powered by a $3 \mathrm{~V}$ lithium battery (CR2247 from Motorola) that allows the device to work for a long time thanks to its high capacity (1000mAh). The electronic circuit of the Bluetooth based temperature and humidity acquisition system is shown in Fig. 2.
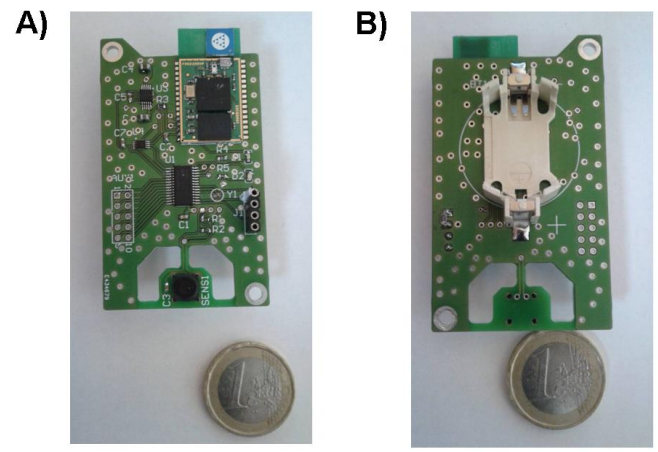

Fig. 2. The electronic circuit of the Bluetooth-based temperature and humidity acquisition system. (A) Top view of the device: the temperature and humidity sensor, the microcontroller, and the Bluetooth module are clearly visible. (B) Bottom view of the device: the battery holder is evident.

\subsection{Multi Sensors Smartphone Application}

In the previous work [1], the smartphone application was developed for communicating directly with a certain sensor, as a prototype of the system. The application read and stored data that aided to analyze them and the system performance and accuracy. The new version of the application reads data from several available sensors that are distributed in short distance around the mobile phone. The user can discover the sensors and select them manually, or this task can be accomplished automatically. The software continuously checks for the availability of the sensors and the connections. Each minute, the application can repeat the process of connection, disconnection, and reading for each sensor. Among each of these processes, the availability of the selected sensors is checked. Also, there is an alarm if no sensor is detected in the network.

The application was developed, as the previous version, for Android mobile phone with the updated version of the Android software development kit (SDK) (with the ADT Plug-in for Eclipse).

The Application called aBluSen has a menu for discovering and selecting desired Bluetooth-based sensors to have environmental information. This part is called discovery. aBluSen distinguishes between non-Sensor Bluetooth-based devices and 
Sensors. Checking the name of the devices is a method to detect differences between Sensors and non-Sensors.

Sensors will be saved as Pair-Sensors after each communication. After selecting sensor/s, the process of connecting starts. In some cases, twice attempts are needed to connect to the sensor. If the standard method of connecting failed, the reflection method starts. The second method avoids failure of connection the standard attempts for connecting. After connecting, the aBluSen tries immediately to read data from the open port. The mobile phone keeps listening to the port to read Input stream while connected. The size of buffer can set from the application that handles the amount of data for each time of reading. The default size is 64 . The obtained data stream needs to follow the process of tokenization to break desired values of temperature and humidity from several lines of data that are read from the sensor. For example, the following line is the sample line of read data for the first attempt to a sensor: $T=+$., +25.6 ,46. Two required values break from the lines. Here +25.6 is the temperature and 46 is the humidity. At the same time, all sensor data stores in a text file in androidbased mobile phone.

The application has a setting to select a number of available sensors to follow mentioned processes automatically. There is a menu with multi-selecting feature to choose sensors then $a B l u S e n$ connects to each of selected sensors as described for the single sensor. The process of reading from connected sensor will be finished after a certain time. This period for each sensor is 1 minute as default. The frequency sample can change for communicating and reading to/from the sensors. After this certain interval, the reading process will be stopped then the application tries to communicate between mobile phone and the other selected sensor. The mobile phone communicates with all sensors and reads and stores information according to the configured interval. As it shown in Fig. 3, the display shows the environmental information and the name of the connected sensor while reading data. The application can work continuously.

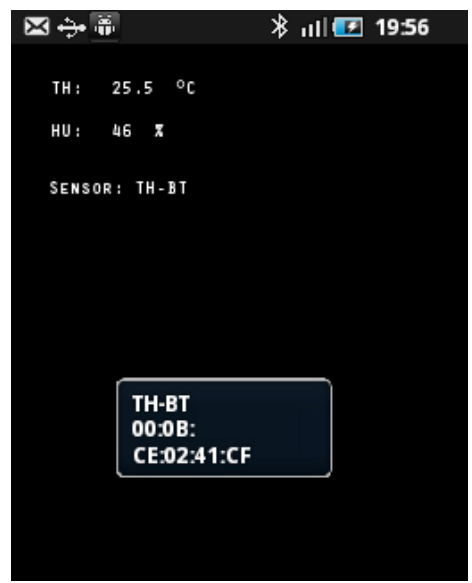

Fig. 3. The interface of the android-based application for acquiring environmental information via Bluetooth 


\subsection{Experiments}

The reliability of the described wireless sensor network was investigated during a first experiment. Three Bluetooth based temperature and humidity acquisition systems were used in the experiment, and the environmental parameters were acquired by the smartphone application. The devices were placed in a climatic chamber (Angelantoni Challenge 250; temperature range for climatic test from $-40^{\circ} \mathrm{C}$ to $+180^{\circ} \mathrm{C}$ ), which allows to control environmental conditions. At the beginning of the experiment the climatic chamber was set with a temperature of $25^{\circ} \mathrm{C}$ and a relative humidity of $50 \%$; this condition was kept for approximately 10 minutes. Then, it was arranged to reach $-20^{\circ} \mathrm{C}$ with a temperature gradient of $-0.5^{\circ} \mathrm{C}$ per minute. The climatic chamber kept the temperature of $-20^{\circ} \mathrm{C}$ for approximately 10 minutes and then it was arranged to go back to $25^{\circ} \mathrm{C}$ with a temperature gradient of $0.5^{\circ} \mathrm{C}$ per minute. At the end of the experiment the climatic chamber kept the temperature of $25^{\circ} \mathrm{C}$ for approximately 10 minutes. During the experiment, the wireless sensor network was set with a sampling frequency of one sample per minute.

The power consumption of the Bluetooth based temperature and humidity acquisition system was measured during a second experiment. The device was powered by a power supply, with a constant voltage of $3 \mathrm{~V}$, and the current was measured using a digital multimeter (34401A from Agilent) with a sampling frequency of 4 samples per second. The device presented in this work was used for the experiment, and the distance between two consecutive temperature and humidity measurements was set to 60 seconds.

\section{$3 \quad$ Results}

Temperature and humidity values, as raw data, acquired using the smartphone application from three Bluetooth-based acquisition systems during the first experiment. The results are shown in Fig. 4. Temperature values obtained by the three devices correctly follow the temperature condition provided by the climatic chamber (see the description in the previous section). The humidity values for temperatures higher than $0^{\circ} \mathrm{C}$ are nearly at $50 \%$ as imposed by the climatic chamber. For temperature lower than $0^{\circ} \mathrm{C}$, since the climatic chamber cannot impose the humidity condition for these temperatures, the humidity values gathered by the three devices are not constant to a fixed value.

The values of the current raw data that are gathered by the Bluetooth based temperature and humidity acquisition system described in this work and the acquired values by the digital multimeter during the second experiment are shown in Fig. 5. The device was connected in series to a digital multimeter, and it was powered by a power supply. The experiment lasted an hour, and the current values shows in Fig. 5 were averaged (since the device transmits the environmental data each minute). The current value absorbed by the device between two transmissions is in the order of 
$1 \mu \mathrm{A}$. The current consumption during the transmission, without any smartphone connected, is in average $8 \mathrm{~mA}$ (Fig. 5A), while during the acquisition with a smartphone is in average $27 \mathrm{~mA}$ (Fig. 5B).
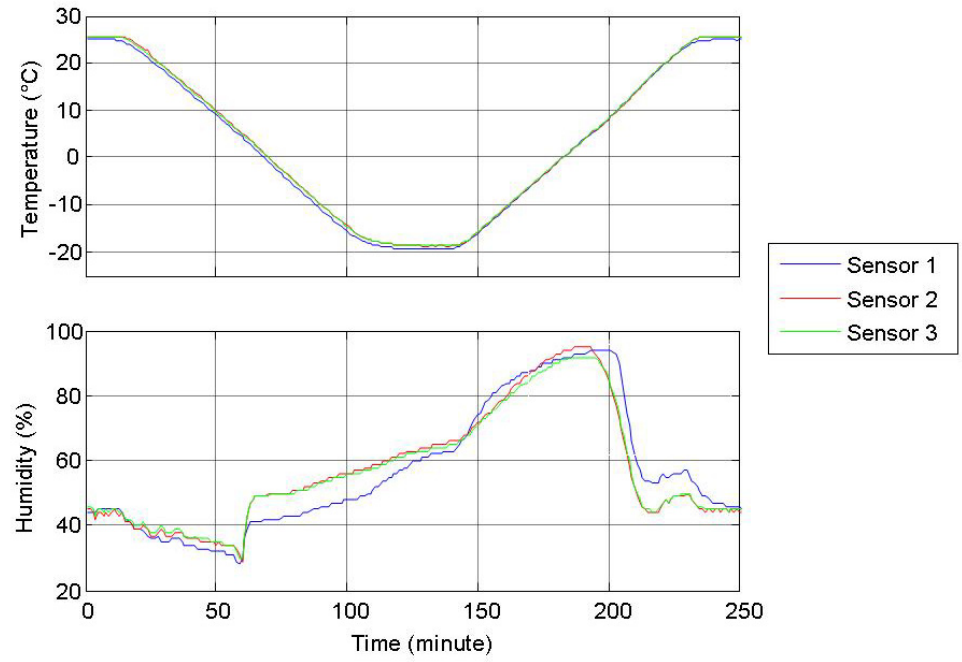

Fig. 4. Temperature and humidity values that are obtained by the three Bluetooth-based temperature and humidity acquisition systems during the first experiment

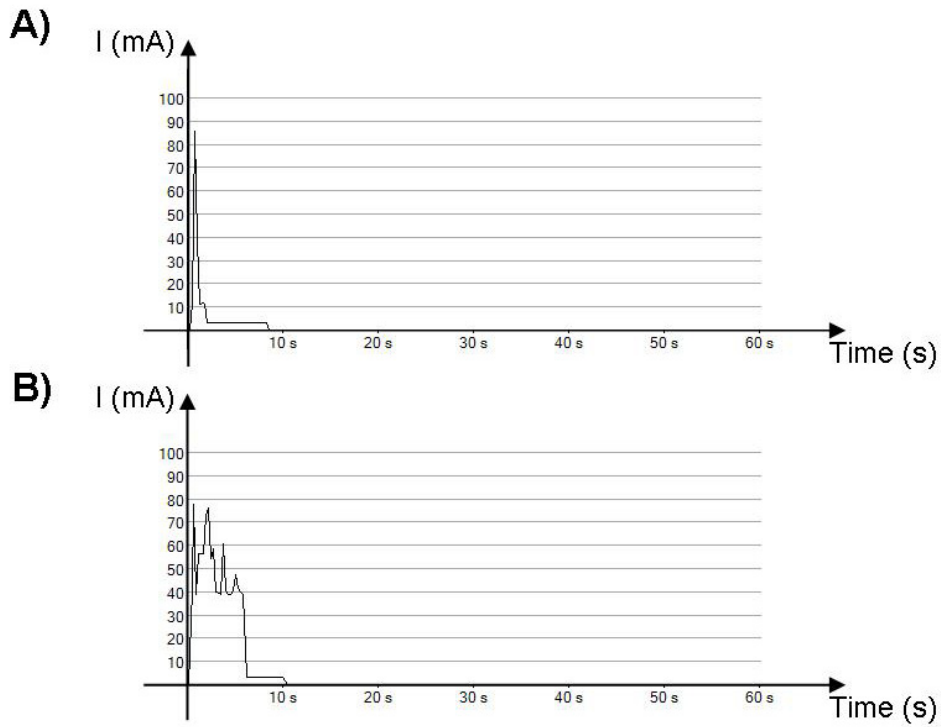

Fig. 5. Current values absorbed by the Bluetooth based temperature and humidity acquisition systems during the second experiment. A) Without smartphone connected. B) With smartphone connected. 


\section{Conclusion and Discussion}

This paper investigates a low power wireless sensor network to acquire temperature and humidity values from a number of devices built using low cost and low power components, and the Bluetooth communication system for the transmission of the acquired environmental data to a smartphone using an application that automatically connects with them.

The performances of the system were tested by a laboratory test using a climate chamber in order to modify the environmental conditions. Results showed that all the devices connected to the wireless sensor network, correctly reported the environmental condition created by the climatic chamber, and the application for the smartphone properly acquired and stored the data sent by the Bluetooth connection. The environmental data was continuously acquired by the smartphone application during the first experiment without any absence of signal from any of the devices that indicate that of the wireless sensor network, is working properly

Results from the second experiment demonstrated that the current consumption of the Bluetooth based temperature and humidity acquisition system is $1 \mu \mathrm{A}$ waiting for a transmission and on average $27 \mathrm{~mA}$ during the transmission to a smartphone. It was calculated that, using a time interval of 15 minutes between two measures and the CR2247 battery, the device can transmit data to a smartphone for more than 4 months.

This approach is useful to monitor climate conditions for small environments, such as laboratories, home rooms, medical spaces, etc., and turn on alarms when the conditions change or go over fixed thresholds. Another possible application of the system presented is the detection of fire in small environments.

Future works will focus on the miniaturization of the device, by using a smaller microcontroller including the radio part of the Bluetooth module, which needs only one external antenna. Other works will focus on reducing the power consumption of the device, modifying the use of the Bluetooth protocol, and choosing a Bluetooth module with a lower current consumption, since about $99 \%$ of the power consumption is caused by the Bluetooth transceiver.

Acknowledgments. This work was sponsored by the Italian private company Reply.

\section{References}

1. Aram, S., Troiano, A., Pasero, E.: Environment sensing using smartphone. In: Sensors Applications Symposium (SAS), Brescia, Italy (2012)

2. Paananen, T.: Smartphone Cross-Platform Framework. Bachelor's Thesis, Jamk University of applied sciences (2011)

3. Firtman, M.: Programming the Mobile Web. O'Reilly Media (2010)

4. Wei, D., Zhu, X., Uchida, M., Ding, S., Cohen, M.: A mobile phone based wearable vital signs monitoring system. In: Fifth International Conference on Computer and Information Technology, Shanghai, China, pp. 950-955 (2005) 
5. Kim, S., Paulos, E.: InAir: sharing indoor air quality measurements and visualizations. In: Proceedings of the 28th International Conference on Human Factors in Computing Systems, New York, pp. 1861-1870 (2010)

6. Staples, E.: EnviSensor: Environmental Data Collection via Participatory Sensor Networks Utilizing Mobile Devices. Master's Thesis, University of Edinburgh (2011)

7. Lee, J., Su, Y., Shen, C.: A Comparative Study of Wireless Protocols: Bluetooth, UWB, ZigBee, and Wi-Fi. In: 33rd Annual Conference on Industrial Electronics Society, pp. 4651. IEEE Press, Taipei (2007)

8. Boudjit, S., Chelghoum, N., Otsmani, M., Allal, S.: Multi-Sensors' Data Gathering Management System for a Wireless Health Monitoring Platform. In: Proceedings of the First ACM MobiHoc Workshop on Pervasive Wireless Healthcare, New York (2011) 